\title{
Displacement Measurements with ARPS in T-Beams Load Tests
}

\author{
Graça Almeida $^{1,2}$, Fernando Melicio $^{2}$, Carlos Chastre $^{1}$, and José Fonseca ${ }^{1}$ \\ ${ }^{1}$ Faculdade de Ciências e Tecnologia, Universidade Nova de Lisboa \\ ${ }^{2}$ Instituto Superior de Engnharia de Lisboa, \\ Lisboa, Portugal \\ mdg.almeida@fct.unl.pt, fmelicio@deea.isel.ipl.pt, \\ chastre@fct.unl.pt, jmf@uninova.pt
}

\begin{abstract}
The measurement of deformations, displacements, strain fields and surface defects in many material tests in Civil Engineering is a very important issue. However, these measurements require complex and expensive equipment and the calibration process is difficult and time consuming. Image processing could be a major improvement, because a simple camera makes the data acquisition and the analysis of the entire area of the material under study without requiring any other equipment like in the traditional method. Digital image correlation (DIC) is a method that examines consecutive images, taken during the deformation period, and detects the movements based on a mathematical correlation algorithm. In this paper, block-matching algorithms are used in order to compare the results from image processing and the data obtained with linear voltage displacement transducer (LVDT) sensors during laboratorial load tests of T-beams.
\end{abstract}

Keywords: Digital Image Correlation, Block Motion Estimation, image processing.

\section{Introduction}

Since the 80's, several works about digital image correlation techniques are under development in order to obtain an accurate knowledge of the displacement field [1-3]. The general idea behind digital image processing techniques is to calculate the displacement field or the strain field without contact and using a simple low cost camera. The research aims to reduce the computation time and to increase the accuracy of the system. When conventional methodology is used the number of the measured points takes a huge importance because they increase the hardware, the time to get the setup ready and the costs. Using image analysis techniques the density of the measured points can be very high. As an example, a trivial image of 1024 by 1024 pixels can be used to obtain a continuous information field with more than 4000 analysis points.

In our previous work[4] the three-step search (TSS) [5] algorithm was studied, especially the simple and efficient (SES) algorithm. A partnership between researchers from the Civil Engineering Department and the Electrical Engineering Department, of the Universidade Nova de Lisboa (UNL) made possible to explore the traditional methodology used in Civil Engineering measurements, which requires a complex and expensive sensorial setup and a very complex calibration process. In this work we compare the results of the traditional approach with the results obtained by digital image processing techniques. 
In this paper the purpose is to use the adaptive rood pattern search (ARPS) and compare it with the data obtained with physical sensors [6]. In this paper only two concrete beams are compared. Although concrete has by itself a good texture a speckle pattern was applied to the T-beams surfaces in order to get an easier image processing (in this paper a random dot pattern was applied). The work developed so far shows that resolution versus specimen dimensions, focal length, distance between camera and specimen, distortion and the speckle pattern are some of the factors that influence the most the measurements error. In order to choose the most promising correlation technique several tests were conducted, such as the comparison of different block matching algorithms and the use of the edge detection techniques. As shown in the previous work, [4] the TSS algorithm shows to be more adequate than edge detection techniques. However, some research questions such as what kind of correlation technique is more efficient or how to increase the accuracy of the system without increasing computation time are still open. Another open issue is what should be the relationship between the entire image and the number of target points. The comparison between the image processing results and the information obtained by the LVDT sensors will be used to establish this relationship.

In [7] a non-touching strain measurement method that covers a pre-defined area is presented. In this work several tests were carried out on RC beams with a span of 4.5 $\mathrm{m}$ and the results were compared to the traditional electrical strain gauges. A speckle pattern correlation was used and a photo was taken every $30 \mathrm{kN}$ until rupture. In this work a camera film was used and the film was scanned with a high quality scanner. The picture was divided into $128 \times 128$ sub-pictures and then subjected to a threshold. The centre of gravity of the resulting black \& white picture was then calculated. With this methodology and the speckle correlation it was possible to find the same subpicture in the second loading condition and therefore estimate the deformation. Despite the good results obtained by this methodology it is significantly dependent on the threshold value and therefore varying with the illumination conditions, speckle pattern and image acquisition parameters. In our work we look for a more stable and conditions independent solution for this problem.

\section{Contributions to Sustainability}

Image processing analysis is used in many situations for earth observations such as resource managements, soils mapping, water resources, etc. The measurements made by image processing simplify the maintenance of local materials, the waste or loss of sensors and human resources are best applied.

With measurements without contact, fewer materials are used which implies reduction of costs and energy. In our tests the same system could analyze several materials with no duplication of the sensors. So it is possible to contribute to a better world with reduction of plastics, iron, and energy.

\section{ARPS - Block Motion Algorithm}

The basic idea of block motion estimation is to divide the current image into a matrix of blocks and then compare these blocks with the previous image in order to calculate 
the displacement vectors. The current block is searched in the previous image in a delimited search area, p pixels around the current block. In our tests a block takes a square side of 16 and the search parameter $p$ is 7.

Block-based motion estimation assumes that objects move in a translational movement. In (Fig. 1) it is possible to see an example of two images that could be compared. At left is the non deformed image and at right the deformed image. It is also possible to see the grid of $16 \times 16$ pixels and the random pattern that was applied. The methodology for estimation of displacement uses the intensity of the pixel and with the unique pattern in each sub-region makes it possible to find the displacement.
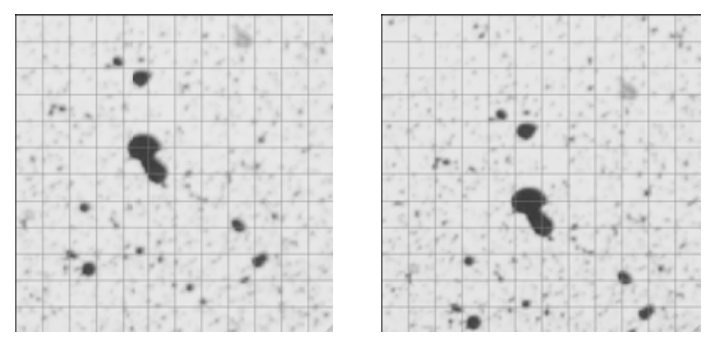

Fig. 1. At left is a no deformed image and at right is the deformed image; images are sub-divide in sub region of $16 \times 16$ pixels

The match block algorithm chosen is based on the least cost. The cost function normally used is the mean square error (MSE) or the mean absolute difference (MAD). In this work the MAD function was used in order to save computation time.

In most cases adjacent blocks have similar motions. The block on the immediate left, above, above-left and above-right of the current block are the most important to calculate the predicted the motion vector (MV) [8]. Four types of region of support could be used (Fig. 2). In this paper the type D was chosen because it requires less memory.
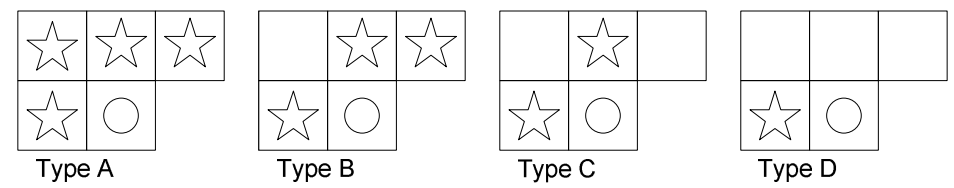

Fig. 2. Regions of support: the blocks market by "O" are on each case the current block and the blocks market with a star are used for predicted the MV

For the initial search, the ARPS algorithm evaluates the four endpoints in a symmetrical rood pattern plus the predicted MV. The four arms of the rood pattern are of equal length (Fig. 3).

The size of the rood pattern is equal to the length of the predicted motion vector (i.e. the motion vector of the immediate left of the current block). In each search points is necessary to compute the MAD function. The size of the rood pattern, $\Gamma$, is calculated in (1), 


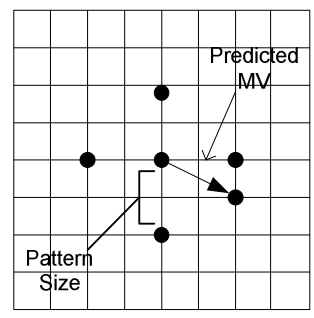

Fig. 3. Symmetrical shape of Adaptive Rood Pattern with four search points locating at the four vertices

$$
\begin{aligned}
\Gamma & =\operatorname{round}\left|\overrightarrow{M V} V_{\text {predicted }}\right| \\
& =\operatorname{round}\left[\sqrt{M V_{\text {predicted }(x)}^{2}+M V_{\text {predicted }(y)}^{2}}\right]
\end{aligned}
$$

The square and the root square operations drawn in (1) require a lot of computation time. Therefore instead of (1) it is possible to use a simplification that only requires the highest magnitude of the two components of the predicted MV (2).

$$
\Gamma=\operatorname{Max}\left\{\left|M V_{\text {predicted }(x)}\right|,\left|M V_{\text {predicted }(y)}^{2}\right|\right\}
$$

When it is not possible to apply the type D of the ROS, the value 2 is chosen for the size of the arm length (i.e. $\Gamma=2$ ). The minimal matching error (MME) point found in the current step will be re-positioned as the new search center of the next search iteration until the MME point is found as the center of the fixed pattern.

\section{Results}

Several load tests have been carried out in order to compare the results obtained by the image processing techniques with the information acquired by a classical measurement system. These tests [6] uses two reference T-beams and three T-beams strengthened with different FRP techniques. In this paper only the results from TSC1 and HB2 are used. The image acquisition conditions for these tests are shown on Table 1. Each image is divided in sub regions of $16 \times 16$ pixels $(\sim 4.4 \mathrm{~mm} \times 4.4 \mathrm{~mm})$.

The TSC1 beam was used as reference without any additional flexural reinforcement and the HB2 beam was reinforced with 3 bonded GFRP sheet layers. The T-beams had a $3 \mathrm{~m}$ span by $0.3 \mathrm{~m}$ heights and were tested until rupture in a 4-point

Table 1. T-Beam image acquisition condition: resolution and the number of photos

\begin{tabular}{ccc}
\hline T beam designation & Resolution & Number of Photos \\
\hline TSC1 & $36 \mathrm{pixel} / \mathrm{cm}$ & 52 \\
$\mathrm{HB} 2$ & $35 \mathrm{pixel} / \mathrm{cm}$ & 27 \\
\hline
\end{tabular}


bending test system. All the tested beams followed a monotonic loading history. The deflection control was granted by seven standard 100mm LVDT, displayed along the longitudinal direction of the beam. At the mid-span, a wire-controlled transducer $(500 \mathrm{~mm})$ was used together with the LVDT $(100 \mathrm{~mm})$ in order to obtain the results of larger deformation, if needed during the post-collapse of the test. The data from the LVDT at the mid-span was used for the comparison with the data obtained with the image system analysis.

The image acquisition for TSC1, and HB2 was done with a digital Cannon EOS $400 \mathrm{D}$ camera with a resolution of $3888 \times 2592$ and two spots of $500 \mathrm{~W}$ each guaranteed artificial light. The artificial lightning was used in order to maintain a constant light environment. All the images were captured on RAW format and then convert to TIFF format for image processing with MATLAB.

Before the data acquisition it was necessary to make the preparation for the image acquisition system (Fig. 4) and the sensor data system acquisition.
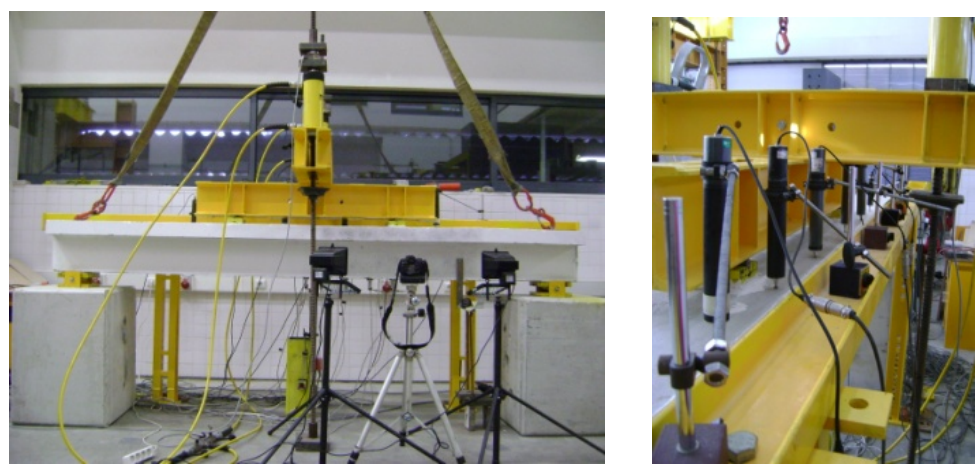

Fig. 4. The preparation of the image acquisition system (left) and data sensor system (right)

The T-beam was initially prepared with an underlying painting of white mate ink and then with a superimposed random speckle pattern manually painted using a large brush with mate black ink. All the digital measurements are done at a distance without any particular or long calibration, having a low cost support, and they are easy to implement in the T-beam test setup.

The photos were taken with intervals of 30 seconds. In first image of the test, the area of interest is over and at the end is down (Fig. 5). In some load tests the displacement could be large.

The digital image processing system compares two adjacent images in order to evaluate the displacement. These results are compared with the results acquired with one of the real sensors used and this evaluation can be seen in a graph of the displacement versus time ( $\mathrm{d} v s . \mathrm{t})$.

In a non-contact strain measurement it is possible to analyze a small area of interest and a large one with the same hardware conditions. Moreover, this technique can be compared with non-linear software based on the finite element analysis (FEA) in order to corroborate the strain fields in the T-beam structure. 

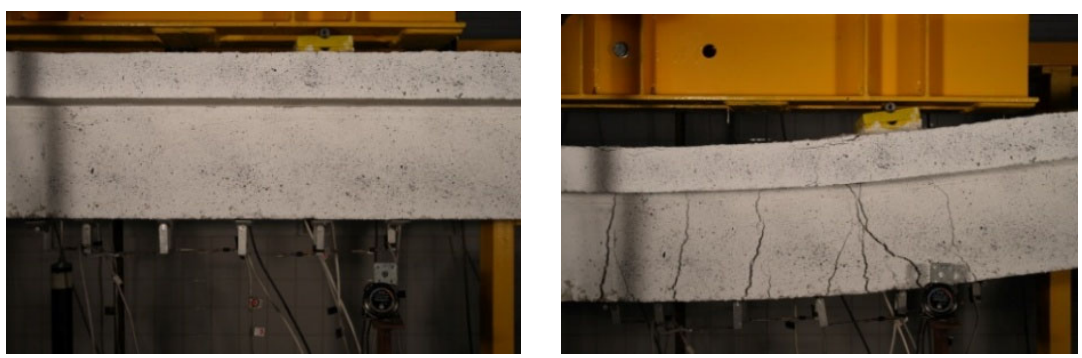

Fig. 5. Images of the test: the first image (left) and the last image (right)
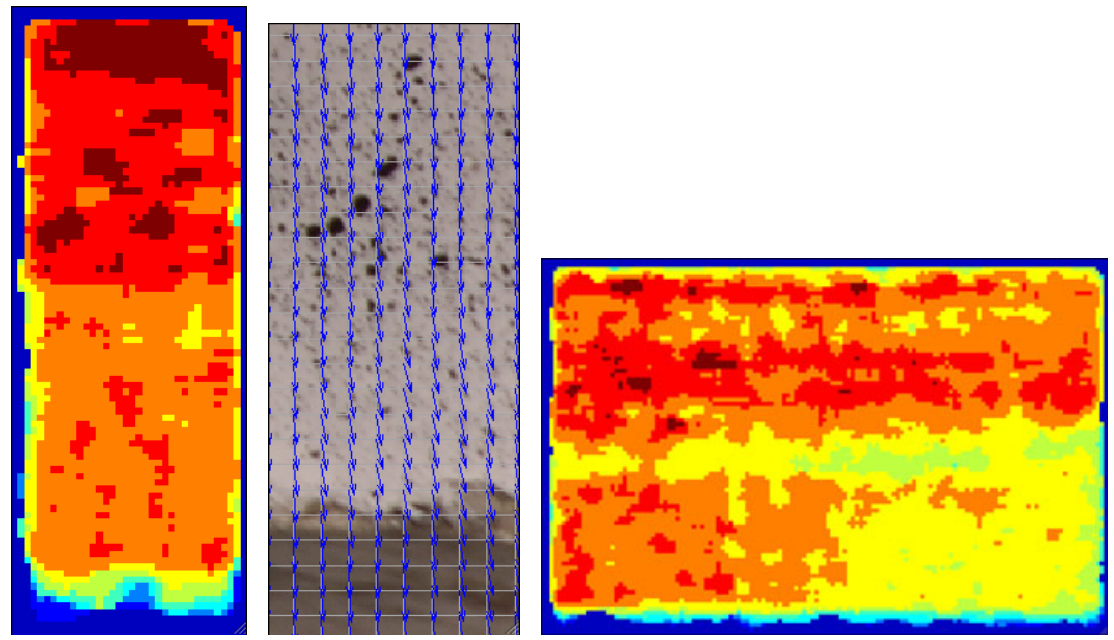

Fig. 6. Representation of displacement map (left) where the dark regions indicated larger displacements, the vector diagram (centre) shows the flow of deformation and with the same system is also possible to compute the complete displacement map (right)

The strain map and the grid vector of the movement vector displacement were done for the small part associated with the LVDT at the mid-span (Fig. 6).

The displacement vector diagram shows the compressive vertical load applied to the specimen. In order to know the implication of the entire T-beam we have also done the strain map of the entire T-beam.

The ARPS algorithm was used in the region of the image associated with the position of the LVDT, for each experiment (TSC1 and HB2), it is possible to visualize the evaluation of the displacement versus time (d vs. t) (Fig. 7).

The abrupt jump indicates that a major crack is found and in this situation the image processing has some problems. The error, before crack situation, was $0.4 \mathrm{~mm}$ for TSC1 and $4.3 \mathrm{~mm}$ for HB2. The error can be reduced if we reduced the interval between photos. 

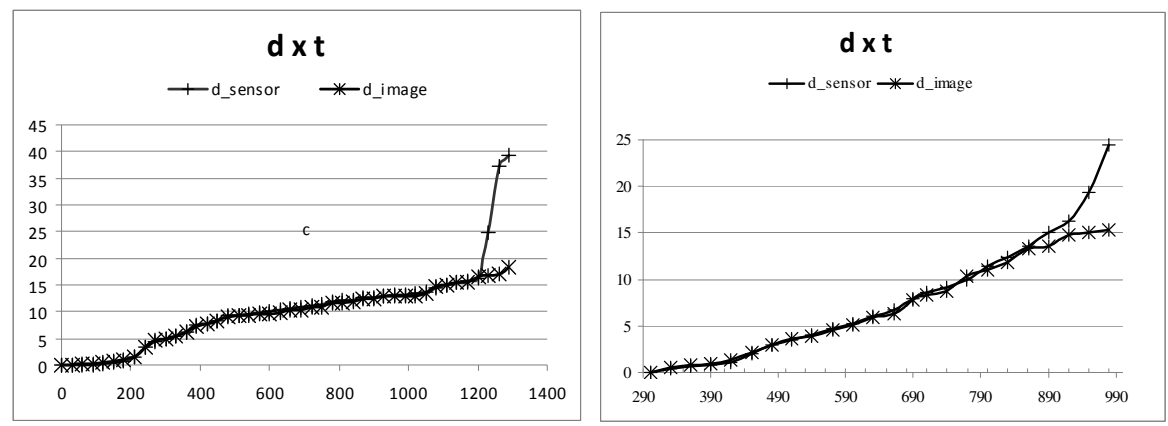

Fig. 7. Evaluation of displacement vs. time with results from image processing (** point) and LVDT sensor (++ point): TSC1 at left and HB2 at right
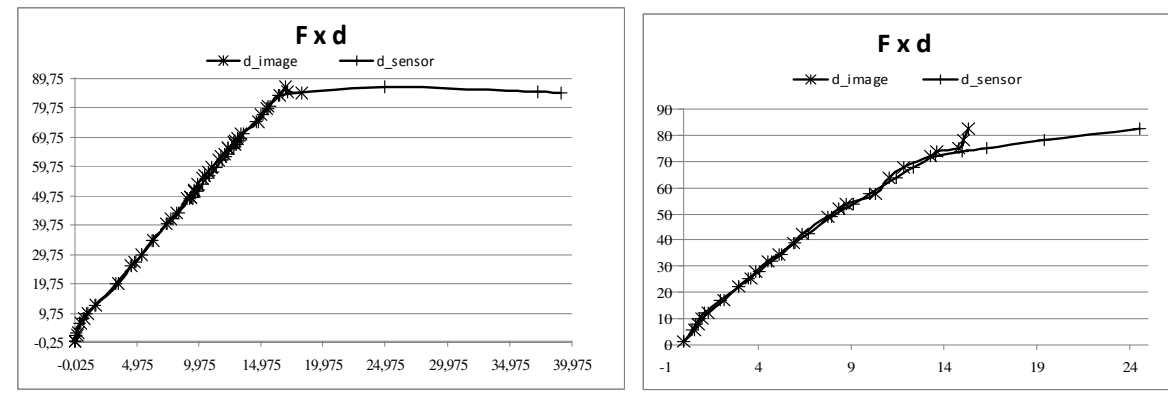

Fig. 8. Evaluation of the force vs. displacement: TSC1 at left and HB2 at right. Values from sensor are market with (++) and image processing with (**).

The evaluation of force versus displacement (F vs. t) is also shown (Fig. 8) and it is also possible to visualize that our system is close to the real sensors.

\section{Conclusions}

The comparison between the measurements from LVDTs sensors and the measurements obtained from image processing techniques revealed to be very similar. Thus, the image processing technique used seems to be a very promising technique for measurement displacements with a much lower investment and much faster and easier setup.

The results obtained in this study shows that it is possible to continue and explore this subject and test the whole area of a T-beam in order to compare with the remain LVDTs sensors. Moreover, a strain field of the T-beam in study can be reproduced and compared to other techniques of modeling structures such as FEA, in order to calibrate and validate the image processor technique. The research will eventually provide software that enables a real time monitoring of the $\mathrm{RC}$ structure during the experimental test. In spite of using median and high pass filter amongst others preprocessing image technique, the results does not show a significant improvement. 
It is important in future work to analyze the influence of the regular pattern and also increase the resolution of the image acquisition in order to obtain more precise results.

\section{References}

1. Chu, T.C., Ranson, W.F., Sutton, M.A., Peters, W.H.: Applications of Digital.ImageCorrelation Techniques to Experimental Mechanics. Experimental Mechanic 25(3), 232-244 (1985)

2. Sutton, M.A., Wolters, W.J., Peters, W.H., Ranson, W.H., McNeill, S.R.: Determination of displacements using an improved digital correlation method. Image and Vision Computing, 133-139 (1983)

3. Peters, W.H., Ranson, W.: Digital Imaging Techniques In Experimental Stress Analysis. Optical Engineering 21(3), 5 (1982)

4. Almeida, G., Biscaia, H., Melicio, F., Chastre, C., Fonseca, J.: Displacement Estimation of a RC Beam Test based on TSS algorithm. In: 5th Iberian Conference on Information Systems and Technologies (CISTI 2010), Santiago de Compostela (2010)

5. Barjatya, A.: Block Matching Algorithms for motion Estimation, DIP 6620 Spring, Final Project Paper (2004)

6. Carvalho, T., Paula, R., Biscaia, C.C.: Flexural Behaviour Of Rc T-Beams Strengthened With Different Frp Materials. In: 3rd Fib International Congress, Washington, D.C (2010)

7. Carolin, A., Olofsson, T., Taljsten, B.: Photographic strain monitoring for civil engineering. In: FRP Composites in Civil Engineering - CICE 2004, Seracino (2004)

8. Yao Nie, K.-K.M.: Adaptive Rood Pattern Search for Fast Block-Matching Motion Estimation. IEEE Transactions On Image Processing 11(12), 8 (2002) 